\title{
Orientierungshilfen in der Corona-Krise - Die Ad-hoc-Empfehlung des Deutschen Ethikrats und die Klinisch-ethischen Empfehlungen von sieben Fachgesellschaften aus der Medizin
}

\author{
Alena Buyx und Volker Lipp
}

\begin{abstract}
Die Corona-Pandemie fordert Gesellschaften und Staaten weltweit in dramatischer Weise heraus. Die Gesundheitssysteme werden in extremer Weise be- und teils auch überlastet. Trotz aller Bemühungen, die Kapazitäten zu erhöhen, stehen mancherorts nicht mehr ausreichend Ressourcen zur Verfügung, um alle Patienten zu behandeln, die einer intensivmedizinischen Behandlung bedürfen. Um den exponentiellen Anstieg der Zahl infizierter und erkrankter Personen und die Ubbrlastung des Gesundheitssystems zu verhindern oder jedenfalls zu verlangsamen, verhängen Staaten flächendeckend massive und rigorose freiheitsbeschränkende Maßnahmen, die das wirtschaftliche und soziale Leben weitgehend zum Erliegen bringen.

Dies führt zu schwerwiegenden Konflikten auf verschiedenen Ebenen. Erstens können Ärzte gezwungen sein zu entscheiden, wer vorrangig intensivmedizinisch behandelt werden soll, wenn die verfügbaren Ressourcen nicht für alle Bedürftigen ausreichen (,Triage-Situationen“). Zweitens müssen einerseits Menschen vor einer Infektion geschützt und bei Erkrankung bestmöglich behandelt und dazu ein hochwertiges und leistungsfähiges Gesundheitssystem verfügbar gehalten und nachhaltig gesichert werden, während andererseits schwerwiegende Folgen für Wirtschaft, Gesellschaft und Bevölkerung, aber auch für den einzelnen möglichst gering zu halten sind.
\end{abstract}

Diese Konfliktlagen sind Gegenstand der hier abgedruckten Ad-hoc-Empfehlung des Deutschen Ethikrats (im Folgenden: ,Ad-hoc-Empfehlung“) und der klinischethischen Empfehlungen von sieben Fachgesellschaften aus der Medizin für Entscheidungen über die Zuteilung von Ressourcen in der Notfall- und der Intensivmedizin im Kontext der COVID-19-Pandemie (im Folgenden: „klinisch-ethische Empfehlungen“). Beide Dokumente unterstreichen übereinstimmend die normative Dimension dieser Konfliktlagen und betonen, dass sie zwar auf medizinisch-wissenschaftlicher Grundlage, aber nicht allein medizinisch zu entscheiden sind, sondern einer fairen und gerechten Abwägung anhand normativ begründeter Kriterien bedürfen. Sowohl die klinisch-ethischen Empfehlungen als auch die Ad-hoc-Empfehlung des Deutschen Ethikrats erörtern die erstgenannte Konfliktlage, nämlich die Triage-Situationen, wenngleich mit unterschiedlicher

Prof. Dr. med. Alena M. Buyx, Technische Universität München, Lehrstuhl für Ethik der Medizin und Gesundheitstechnologien, Mitglied des Deutschen Ethikrats, Peer Reviewer und Beraterin der Schreibgruppe der Klinisch-ethischen Empfehlungen, Ismaninger Str. 22, 81675 München, Deutschland

Prof. Dr. iur. Dr. h.c. Volker Lipp, Universität Göttingen, Lehrstuhl für Bürgerliches Recht, Zivilprozessrecht, Medizinrecht und Rechtsvergleichung,

Mitglied des Deutschen Ethikrats,

Platz der Göttinger Sieben 6, 37073 Göttingen, Deutschland
Akzentuierung. Die Ad-hoc-Empfehlung des Deutschen Ethikrats geht darüber hinaus auch auf die zweite, gesamtgesellschaftliche Konfliktlage ein. Bereits hieran wird die unterschiedliche Ausrichtung und Zielsetzung beider Dokumente deutlich. Sie entspricht der unterschiedlichen Funktion und Legitimation ihrer jeweiligen Verfasser.

Die klinisch-ethischen Empfehlungen sind von Autoren und Experten aus sechs medizinischen Fachgesellschaften und aus der Akademie für Ethik in der Medizin erarbeitet worden. Vorbereitend waren Impulse aus der aktuellen klinisch-ethischen Beratungspraxis in Videokonferenzen mit Hunderten Teilnehmern gesammelt worden. Eine interdisziplinär besetzte Schreibgruppe entwickelte dann einen Entwurf der Empfehlungen, der einem Peer Review unterzogen wurde. Die danach veröffentlichten Empfehlungen sollen die verantwortlichen Akteure bei ihren Entscheidungen in der klinischen Praxis unterstützen, wer vorrangig intensivmedizinisch behandelt werden soll. Dazu entwickeln sie medizinisch und ethisch begründete Kriterien und Verfahrensweisen. Sie sind - wie andere derartige Empfehlungen - durch den Status ihrer Verfasser als Fachgesellschaften legitimiert. Die Empfehlungen werden gegenwärtig in Reaktion auf Impulse aus der Praxis bereits wieder überarbeitet; daher werden sie hier nicht abgedruckt. Die jeweils aktuelle Fassung findet sich hier: www.divi.de.

Der interdisziplinär, interprofessionell und pluralistisch zusammengesetzte Deutsche Ethikrat bearbeitet gemäß seinem gesetzlichen Auftrag ( $\mathbb{2}$ EthRG) ethische, gesellschaftliche, naturwissenschaftliche, medizinische und rechtliche Fragen sowie die voraussichtlichen Folgen für Individuum und Gesellschaft, insbesondere auf dem Gebiet der Lebenswissenschaften und ihrer Anwendung auf den Menschen. Zu seinen Aufgaben gehören u. a. die Information der Öffentlichkeit und die Förderung der Diskussion in der Gesellschaft, die Erarbeitung von Stellungnahmen sowie von Empfehlungen für politisches und gesetzgeberisches Handeln. Seine Ad-hoc-Empfehlung will daher primär Politik und Gesellschaft für die normative Dimension der Konfliktlagen sensibilisieren. Sie enthält Kriterien für die Überprüfung der aktuellen Infektionsschutzmaßnahmen und entwickelt Empfehlungen für Öffnungsperspektiven und Wege, wie in verantwortlicher Weise zu einer „Re-Normalisierungsstrategie“ übergegangen werden kann, in der schrittweise Einschränkungen des wirtschaftlichen, gesellschaftlichen, politischen und privaten Lebens zurückgenommen werden können.

Darüber hinaus soll die Ad-hoc-Empfehlung Orientierungshilfe in den Triage-Situationen leisten, indem sie den grundlegenden ethischen und rechtlichen Rahmen beschreibt und die Bedeutung der ärztlichen Selbstregulierung, etwa in Form der hier abgedruckten klinisch-ethischen Empfehlungen, beleuchtet. Die Ad-hoc-Empfehlung des DER will daher die konkreten handlungsleitenden kli- 
nisch-ethischen Empfehlungen nicht ersetzen - und könnte es auch nicht. Beide Dokumente dürfen, ja müssen jedoch hinsichtlich der Triage-Situationen auf einander bezogen gelesen werden. Wir freuen uns, dass beide Dokumente in diesem Heft aus rechtlicher und aus ethischer Sicht analysiert und kommentiert werden.

Aus rechtlicher wie aus ethischer Sicht unter allen schwierigen Konstellationen wohl die problematischste ist vermutlich die Ex-post-Triage, also etwa das Abhängen einer multimorbiden Patientin von einem lebensnotwendigen Respirator (der bei unverändertem Therapieziel weiterhin indiziert und vom Patientenwillen getragen wäre) aus Gründen der Ressourcenknappheit zugunsten eines Patienten mit deutlich besserer Prognose. Die klinisch-ethischen Empfehlungen wollen diese Situation gleich behandeln wie die Ex-ante-Triage, in der es um die Frage geht, welcher von mehreren Patienten den einzigen verfügbaren Respirator bekommt. Für beide Konstellationen ist nach der initialen bzw. kontinuierlichen Behandlungsnotwendigkeit die klinische Erfolgsaussicht das entscheidende Kriterium. Allerdings betonen die klinisch-ethis chen Empfehlungen, ihre Aussagen beruhten auf den nach Einschätzung der Verfasser am ehesten begründbaren ethischen Grundsätzen in einer tragischen Entscheidungssituation. Eine abschlieBende juristische Einordnung sei nicht Gegenstand dieser Empfehlungen. Die Rechtslage bleibt deshalb dort letztlich offen. Die Ad-hoc-Empfehlung des Deutschen Ethi- krats meint erstens, dass diese beiden Situationen ethisch wie rechtlich nicht gleich bewertet werden können, geht zweitens etwas genauer auf die Rechtslage ein und meint drittens, dass die Beendigung einer bereits laufenden Therapie zugunsten eines Patienten mit besserer Prognose auf Grundlage der Rechtsprechung des BVerfG und des BGH nicht gerechtfertigt werden, d.h. keine allgemeine Regel bilden könne. Einem Arzt, der in einem solchen Entscheidungskonflikt steht, dürfe allerdings kein Schuldvorwurf gemacht werden, wenn er sich z. B. nach den klinisch-ethischen Empfehlungen richtet und Patientin mit schlechterer Prognose vom Respirator nimmt und diesen für den $\mathrm{Pa}-$ tienten mit besserer Prognose verwendet.

Die in beiden Dokumenten und den jeweiligen Kommentaren verhandelten Themen gehören ohne Zweifel zu den besonders herausfordernden normativen Problemstellungen für moderne Gesellschaften und ihre Gesundheitssysteme. Erste Antworten sind unter großer Zeitnot und gleichzeitiger empirischer Ungewissheit zu Covid-19 entstanden. Die Texte markieren den Beginn des aktuellen Denk- und Diskussionsprozesses in den normativen Wissenschaften zum Umgang mit Verantwortung und Solidarität in einem freiheitlichen, auf der Anerkennung der Menschenwürde und der Grundrechte basierenden Gemeinwesen unter Krisenbedingungen. Wir hoffen, dass sie diesen dringend notwendigen Debatten als Grundlage dienen und die wissenschaftlichen wie öffentlichen Diskurse bereichern.

\section{Orientierung in der Corona-Krise? Nicht mit Doppelbotschaften}

\section{Weyma Lübbe}

\begin{abstract}
Der folgende Beitrag analysiert Unstimmigkeiten in der Ad hocEmpfehlung ,Solidarität und Verantwortung in der Corona-Krise" des Deutschen Ethikrats und in den klinisch-ethischen Empfehlungen „Entscheidungen über die Zuteilung von Ressourcen in der Notfall- und der Intensivmedizin im Kontext der COVID19-Pandemie" der einschlägigen medizinischen Fachgesellschaften. Es wird gezeigt, dass die Orientierungsfunktion solcher Stellungnahmen leidet, wenn grundlagentheoretische Probleme, die innerhalb der Normwissenschaften kontrovers sind, in den Texten versteckt anstatt gezielt thematisiert werden. Um normative Orientierung in der Corona-Krise zu leisten, ist der Diskussionsstand in den beteiligten Disziplinen derzeit nicht ausreichend integriert.
\end{abstract}

\section{Versteckte Kontroversen}

Der folgende Beitrag möchte zur vertieften Auseinandersetzung mit der am 27.3.2020 herausgekommenen Ad hoc-Empfehlung ,Solidarität und Verantwortung in der Corona-Krise" des Deutschen Ethikrats (S. 466 in diesem Heft) und den kurz zuvor, am 25.3., von medizinischer Seite publizierten klinisch-ethischen Empfehlungen „Entscheidungen über die Zuteilung von Ressourcen in der Notfall- und der Intensivmedizin im Kontext der COVID-

Prof. Dr. phil. Weyma Lübbe,

Institut für Philosophie, Universität Regensburg,

93040 Regensburg, Deutschland
19-Pandemie" ${ }^{\text {"1 }}$ beitragen. Ich beschränke mich dabei auf die Frage der Bewältigung dilemmatischer, knappheitsbedingter Entscheidungssituationen in der medizinischen Versorgung von COVID-19-Patienten, die in beiden Stellungnahmen thematisiert wird.

Beide Dokumente sind angesichts der Kürze der Erstellungszeit und der Rücksichten, die bei kollektiver Autorschaft zu nehmen sind, bemerkenswert differenziert und konsensuell geraten. Sie entsprechen damit dem Auftrag, der solchen Stellungnahmen im Allgemeinen zugeschrieben wird. Es handelt sich um Versuche, den betroffenen Praktikern und der gesamten Öffentlichkeit Orientierungshilfen zu geben, und zwar in diesem Fall inmitten einer Lage, die für moderne Gesellschaften präzedenzlos ist. Viele der sofort zu gebenden Antworten auf die Corona-Krise waren und sind weder in den Gesetzestexten noch in den Schubladen von Normwissenschaftlern vorhanden. Über sie muss inmitten der Krise entschieden und auch inmitten ihrer reflektiert und diskutiert werden. Das ist ein Findungsprozess, der notgedrungen tentativ ist. Beide Empfehlungstexte lassen diese Einschätzung selbst erkennen. Und beide lassen auch die Einsicht erkennen, dass zu den anstehenden Fragen Vertreter mehrerer Disziplinen zu hören sind.

So gut wie gar nicht lassen die beiden Dokumente erkennen, wie tiefsitzend, wie alt (ideengeschichtlich betrachtet) und vor allem: wie kontrovers die theoretischen

1) Zugänglich über www.divi.de, Abruf am 5.4.2020. 